\title{
Characterization of Hot-Compressed Magnesium Alloys in a Scanning Electron Microscope
}

\author{
Shirin Kaboli, Hendrix Demers, and Raynald Gauvin
}

Department of Mining and Materials Engineering, McGill University, Montréal, Canada.

Under specific operation conditions in a scanning electron microscope (SEM), the secondary electron (SE) and backscattered electron (BSE) contrast include a contribution from the changes in crystallographic orientation of the bulk specimen. The crystallographic contrast is about $5 \%$ of the total signal and superimposed on the normal topographical and compositional contrast. In a non-deformed polycrystalline microstructure, the crystallographic contrast is commonly observed between grains of different crystal orientation, known as grain contrast or simply orientation contrast. In a deformed polycrystalline microstructure, local crystallographic contrast is observed inside each grain, indicating the variation of crystal orientation due to deformation. The variation of crystal orientation can be random or regular. If a deformed grain contains a mosaic structure of sub-grains with random crystal orientation relative to the nominal crystal orientation of the parent grain, a random crystallographic contrast is observed inside the grain. This contrast is known as sub-grain contrast since it is attributed to the presence of misoriented sub-grains inside the high angle grain boundaries of a deformed grain. If deformation occurs in a progressive manner inside a grain, the variation of crystal orientation is not random. As a result, a regular crystallographic contrast is observed inside the grain. This contrast is known as bend contour contrast due to the crystallographic origin and similar appearance to the bend contours observed in micrographs of bent thin foils in a transmission electron microscope [1]. In this study, the effects of SEM operation conditions were studied on the appearance of bend contour contrast in a hot-compressed Magnesium (Mg) alloy specimen.

The Mg-0.2Al-0.3Ca (wt $\%$ ) alloy was selected for the uniaxial hot-compression testing at a temperature of $400{ }^{\circ} \mathrm{C}$, a strain rate of $0.01 \mathrm{~s}^{-1}$, and a strain of 0.6 using a $100 \mathrm{kN}$ servo-hydraulic materials testing system. The BSE imaging was carried out at $1-3^{\circ}$ specimen tilt range, a 10 and $30 \mathrm{keV}$ electron beam energy and a $7 \mathrm{~mm}$ working distance using a Hitachi SU-8000 cold-field emission SEM. The electron backscatter diffraction (EBSD) crystal orientation mapping was carried out at $70^{\circ}$ specimen tilt, a 20 $\mathrm{keV}$ electron beam energy and a $25 \mathrm{~mm}$ working distance.

Fig. 1 shows the microstructure of the deformed specimen after the uniaxial hot-compression test. Fig. 1a shows the BSE micrograph of the area inside an elongated deformed Mg grain. A large and nonuniform bend contour contrast in the form parallel contours was observed inside this grain. In addition, two horizontal scratches from the mechanical grinding and polishing steps were indicated with red arrows. Using the different SEM operation conditions such as stage tilt and electron beam energy, a number of examinations were carried out to study the origin of the parallel contours of contrast observed in Fig. 1a and differentiate them from the internal deformation substructures such as low angle boundaries. Fig. 1a-c show the BSE micrographs obtained at $1^{\circ}, 2^{\circ}$, and $3^{\circ}$ stage tilt and a beam energy of $30 \mathrm{keV}$, respectively. Fig. 1d-f show the BSE micrographs obtained at $1^{\circ}, 2^{\circ}$, and $3^{\circ}$ stage tilt and a beam energy of $10 \mathrm{keV}$, respectively. For stage tilt examinations, the offset of the electron beam axis versus stage tilt center was corrected for a eucentric tilt. The position, width, and BSE intensity of contours varied when the stage was tilted. Also, the position of contours remained constant with a change of the electron beam energy. However, the width and BSE intensity of the contours varied with a 
change of the electron beam energy. The width of contours decreased with an increase in electron beam energy. These examinations clearly indicated the dependence of the contours to the local crystal orientation relative to the electron beam. Furthermore, the inverse pole figure (IPF) coloring map of the same area is displayed in Fig. 1g. Color variation associated with crystal misorientation and low angle grain boundaries were observed in this grain. These results show that bend contour contrast in the BSE micrograph is related to the local crystal misorientation (i.e. rotation of the Mg crystal across the grain) during deformation [2].

\section{References:}

[1] D C Joy in "Quantitative scanning electron microscopy", ed. D. B. Holt, (Academic Press, London), pp. 131-181.

[2] S Kaboli, H Demers, N Brodusch and R Gauvin, J. Appl. Crystallogr. (2014) Submitted.

[3] The authors thank Dr. S Zaefferer at Max-Planck Institute for Metals Research (MPIE) for his technical assistance and contributions to this work.
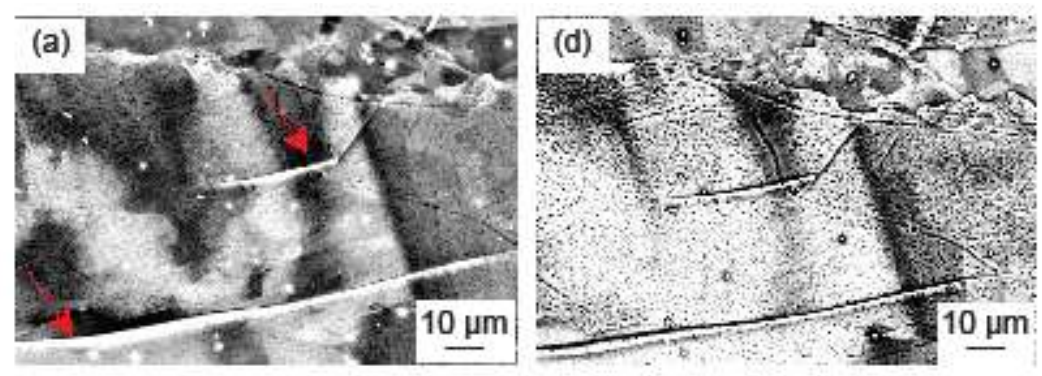

(g)


Figure 1. The microstructure of $\mathrm{Mg}-0.2 \mathrm{Al}-0.3 \mathrm{Ca}(\mathrm{wt} \%)$ alloy after the uniaxial hot-compression test. Backscattered electron micrographs obtained (a) $1^{\circ}$ (b) $2^{\circ}$ (c) $3^{\circ}$ at a beam energy of $30 \mathrm{keV}$ and (d) $1^{\circ}$ (e) $2^{\circ}$ (f) $3^{\circ}$ at a beam energy of $10 \mathrm{keV}$. (g) an inverse pole figure coloring map of the same area. The position, width and BSE intensity of contours varied when the stage was tilted. The width and BSE intensity of the contours varied with a change of the electron beam energy. The appearance of the contours of contrast was related to the rotation of the $\mathrm{Mg}$ crystal across the grain during deformation. 